\title{
Clinical evaluation of a new orthosis, the 'Walkabout', for restoration of functional standing and short distance mobility in spinal paralysed individuals
}

\author{
JW Middleton, JD Yeo, L Blanch, V Vare, K Peterson and K Brigden \\ Moorong Spinal Injuries Unit, Royal Rehabilitation Centre, Sydney, Australia
}

\begin{abstract}
The Walkabout orthosis is a relatively new device for assisted standing and mobility in spinal paralysed individuals. The design, with a medially-mounted single-axis hinge joint linking two knee-ankle-foot orthoses, is quite different to other currently available orthoses which have laterally positioned hip joints such as the Reciprocal Gait Orthosis or Hip Guidance Orthosis. Twenty-five spinal cord injured patients were fitted and trained with the Walkabout orthosis and followed up regularly for just under 2 years on average. Sixty percent of all the patients fitted have incorporated use of the Walkabout orthosis into their lifestyles. Maintenance of joint mobility and psychological benefits were the most important outcomes of Walkabout usage. Loss of thoraco-lumbar mobility was found to be a limiting factor in successful use of the Walkabout orthosis in patients without active hip flexion. Patient selection criteria should include demonstrated spinal stability without significant deformity, controlled muscle spasm, less than $5^{\circ}$ of hip or knee flexion contracture, achievable neutral ankle position, mobility of the thoraco-lumbar spine into lateral flexion, good upper limb strength, and motivation with realistic expectations.
\end{abstract}

Keywords: spinal cord injury; orthosis (Walkabout); standing; mobility; outcomes

\section{Introduction}

Benefits of standing and ambulation with orthoses for paralysed individuals suggested in the literature have included reduction of osteoporosis and subsequent fracture, reduction of hypercalciuria and urinary calculi formation, reduction of heterotopic ossification, reduction in spasticity, maintenance of joint range of motion and improvement in psychological wellbeing. ${ }^{1-6}$ Recently several authors have suggested that at least in the short term, the physiological benefits to paraplegic individuals of regular standing and some walking in orthoses may not be as great as previously claimed. ${ }^{7,8}$

Although orthoses have enabled some individuals to overcome architectural barriers such as stairs or narrow doorways, ${ }^{5}$ in practice most patients have found that the necessary accompanying walking aids interfere with task performance and that the wheelchair is more efficient for mobility. ${ }^{9,10}$ Even so, a large proportion of paraplegic individuals still desire improved mobility to overcome the problems of inaccessibility. ${ }^{11}$ In contrast to an earlier study reporting that braces prescribed during rehabilitation are frequently later abandoned, particularly in patients with a neurological lesion above $\mathrm{T} 12,{ }^{12}$ several authors

Correspondence: JW Middleton have now reported an encouraging trend towards continued long-term usage of orthoses for exercise with patients describing strong feelings of physical and psychological well-being associated with their use. ${ }^{9,13}$

Over the last $10-15$ years there has been renewed interest internationally in ambulation of paraplegic individuals with the attempted development of more mechanically efficient orthoses, experimentation with electrical stimulation ${ }^{14}$ and recently several hybrid systems combining a mechanical orthosis with electrical stimulation. ${ }^{15,16}$ Orthotic devices allowing paralysed individuals to stand and ambulate reciprocally include the Hip Guidance Orthosis (HGO) ${ }^{17}$ the Louisiana State University-Reciprocating Gait Orthosis (LSU-RGO), ${ }^{18}$ the modified Douglas RGO, ${ }^{16}$ the Advanced Reciprocating Gait Orthosis (ARGO), ${ }^{19}$ the Isocentric $\mathrm{RGO}^{20}$ and the Walkabout orthosis. ${ }^{21,22}$ The Walkabout device designed by Polymedic Pty Ltd, Queensland, Australia is a modular orthotic component which attaches to locked knee, rigid ankle, cosmetic orthoses (KAFO's) to form a medial linkage joint. The concept of medially-linking KAFO's has been previously described both alone and in combination with electrical stimulation. ${ }^{23,24}$

The purposes of this clinical study were:

(1) To evaluate the Walkabout orthosis for restoration of functional standing and short distance mobility in spinal cord injured (SCI) individuals. 
(2) To establish criteria for prescription of the Walkabout orthosis and define its potential role in the rehabilitation and lifestyle of SCI individuals.

\section{Methods}

The 'Walkabout' program was established at the Moorong Spinal Unit, Royal Rehabilitation Centre, Sydney, in August 1992, to assess the Walkabout orthosis shown in Figure 1.

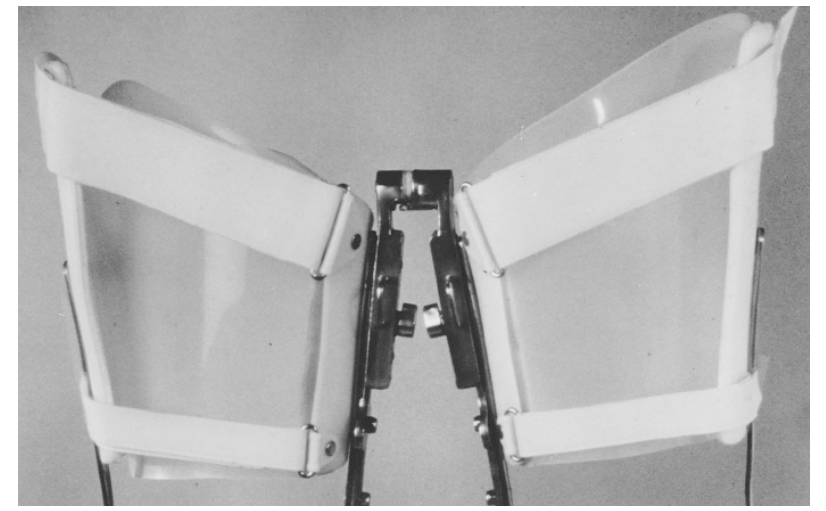

Figure 1 The Walkabout device medially linking two kneeankle-foot orthoses

\section{Subjects}

Twenty-five adult individuals with spinal cord injuries who were fitted and trained in the Walkabout orthosis prior to home use have been followed up regularly since they commenced the program. Patient profiles are summarised in Table 1 . Nineteen of the 25 patients were male and six were female with a mean age and standard deviation in all patients of $35 \pm 13$ years and a range from 20 to 71 years. The majority of patients had suffered traumatic injuries. Six patients were paralysed due to non-traumatic causes including complications of post-spinal surgery (three), spinal tumour (two) and post-viral (one). The interval between onset of paralysis and fitting of the Walkabout ranged from $4 \frac{1}{2}$ months to 20 years.

\section{Inclusion criteria}

Prior to acceptance into the program, each patient underwent a multi-disciplinary assessment by a medical specialist, a physiotherapist and an orthotist. Patient lifestyle, expectations and motivation were assessed by clinical interview for suitability to participate. Physical examination confirmed neurological level of lesion and degree of impairment, presence or absence of contractures and degree of spasticity. Inclusion criteria were motivation with realistic expectations, absence of clinically significant joint contractures, functional upper limb strength and general fitness and ability to

Table 1 Summary of individual subject profiles

\begin{tabular}{|c|c|c|c|c|c|c|}
\hline Patient & Sex & $\begin{array}{c}\text { Age } \\
\text { (years) }\end{array}$ & $\begin{array}{l}\text { Neurological } \\
\text { Impairment* }\end{array}$ & $\begin{array}{c}\text { Duration of } \\
\text { paralysis } \\
\text { (years) }\end{array}$ & $\begin{array}{l}\text { Previous } \\
\text { orthosis }\end{array}$ & $\begin{array}{c}\text { Continuing } \\
\text { usage }\end{array}$ \\
\hline 1 & M & 26 & T6 Comp & 1.8 & KAFO’s (gait) & Yes \\
\hline 2 & M & 51 & T12 Comp & 3.8 & KAFO's (stand) & Yes \\
\hline 3 & $\mathrm{~F}$ & 20 & C6 Inc (C) & 1.2 & Backslabs & Yes \\
\hline 4 & M & 22 & T10 Comp & 1.5 & Backslabs & No \\
\hline 5 & $\mathrm{~F}$ & 32 & T12 Inc $(\hat{C})$ & 2.5 & Backslabs & Lost to follow-up \\
\hline 6 & $\mathrm{~F}$ & 26 & T9 Inc (C) & 10.9 & None for years & Yes \\
\hline 7 & M & 32 & T12 Comp & 9.8 & Backslabs & No \\
\hline 8 & M & 60 & T7 Inc (C) & 3.5 & KAFO's (stand) & No \\
\hline 9 & M & 21 & T6 Comp & 3.5 & Backslabs & Yes \\
\hline 10 & $\mathrm{~F}$ & 42 & $\mathrm{~T} 2 \operatorname{Inc}(\mathrm{C})$ & 0.6 & Backslabs & Yes \\
\hline 11 & M & 44 & T5 Inc (C) & 1.2 & KAFO's (gait) & No \\
\hline 12 & M & 50 & T4 Comp & 6.3 & Never stood & No \\
\hline 13 & M & 42 & T8 Comp & 16.5 & Backslabs & Yes \\
\hline 14 & M & 71 & T12 Inc (C) & 5.5 & KAFO's (stand) & Yes \\
\hline 15 & M & 25 & T10 Comp & 1.3 & Backslabs & No \\
\hline 16 & M & 40 & T6 Inc (C) & 18.0 & Stood on spasm & Yes \\
\hline 17 & M & 34 & T9 Comp & 2.9 & Backslabs & Yes \\
\hline 18 & M & 30 & C5 Inc (C) & 0.5 & Backslabs & Yes \\
\hline 19 & M & 25 & T10 Comp & 0.4 & Backslabs & No \\
\hline 20 & $\mathrm{~F}$ & 21 & T10 Inc $(\hat{C})$ & 1.8 & KAFO's (gait) & Yes \\
\hline 21 & M & 30 & T8 Comp & 5.0 & Never stood & No \\
\hline 22 & M & 24 & T12 Inc (C) & 0.6 & None (rehab) & Yes \\
\hline 23 & $\mathrm{~F}$ & 31 & T10 Comp & 11.0 & None for years & Yes \\
\hline 24 & M & 31 & T8 Comp & 5.0 & KAFO's (stand) & Yes \\
\hline 25 & M & 45 & T7 Comp & 20.0 & None for years & Yes \\
\hline
\end{tabular}

*ASIA Impairment Classification listed in parentheses for incomplete lesions. $\uparrow$ Time from injury to commencement 
stand without back pain. The expected commitment necessary for participation in the program and potential risks were discussed and informed consent was then obtained.

\section{Principles of preambulatory and gait training}

The training program initially concentrated on gaining hands-free standing. Gait training was then commenced in parallel bars with progression to using a rollator frame or elbow crutches when appropriate. Specific training in donning/doffing of the orthosis and transfers between sitting and standing was necessary in all patients. Orthotic modifications were considered at every stage during the training process. Regular outpatient follow-up appointments were made for upgrading of skills. Typically, between 10-15 working days were required to progress through the stages of gait training from balanced standing, transfers, ambulation in parallel bars, use of crutches or a frame outside of parallel bars and finally to attempting small gradients and different types of surface.

\section{Measurement of outcome}

A detailed questionnaire was administered at outpatient review or by telephone interview to all patients who successfully completed gait training and continued to use the Walkabout. All patients who have continued to use the Walkabout for 18 months or more were interviewed at least twice during this period, the first time between 7-12 months and the second time approximately 12 months later. Five of the patients who discontinued using the Walkabout were interviewed after withdrawing from the program.

\section{Statistical analysis}

Analysis of variance of differences in the pattern of orthotic usage between complete and incomplete SCI individuals was performed using the SPSS $^{\circledR}$ for Windows ${ }^{\circledR}$ statistical package (Version 6). An alpha level of $P \leqslant 0.05$ was chosen a priori to determine statistical significance.

\section{Results}

Sixteen of the original 25 patients who commenced in the study still use the Walkabout, with 15 of these having continued for more than 18 months. One patient was lost to follow-up. All but one of the 25 patients, a woman with incomplete tetraplegia (patient 3 ), were able to apply the Walkabout orthosis independently and transfer themselves between standing and sitting whilst wearing it. Several others with high levels of lesion preferred to seek assistance to don and doff the orthosis.

Frequency, duration and intensity of walkabout usage The frequency, duration and total intensity (frequency $x$ duration) of orthosis usage per week for both complete and incomplete SCI individuals are shown in Table 2. No statistically significant differences between the two groups were found to exist for any variable of orthotic usage at either first or second review (all $P>0.05)$. Similarly, no significant differences in variables within groups were found over time. The mean intensity of Walkabout usage and standard error for both groups combined was $150 \pm 24 \mathrm{~min}$ and $169 \pm 36 \mathrm{~min}$ with a median of $120 \mathrm{~min}$ each at first and second review respectively.

\section{Walking aids}

Although no significant differences in usage patterns existed between groups of complete and incomplete SCI individuals, significant differences in preference of walking aid were noted. The majority of complete SCI individuals in this study preferred to continue ambulating indoors at home using parallel bars for support due to the stability, safety and ease of initial transfer from sitting to standing that they offered. Whilst the majority of incomplete SCI individuals ambulated outdoors using forearm crutches or a frame. However, several individuals with complete lesions between the T10-12 level did ambulate proficiently over even ground using a rollator frame. One other individual with a complete T6 lesion who could also ambulate well over short distances using forearm crutches reverted to ambulating in parallel bars after losing confidence following a fall.

\section{Functional performance using the Walkabout}

Activities performed in standing by individuals with complete lesions using the Walkabout included food preparation and cooking, washing dishes, defrosting a refrigerator freezer, taking linen out of a cupboard, reaching objects in the top of a wardrobe, reaching

Table 2 Summary of pattern of usage (mean \pm SEM) of the Walkabout orthosis for complete and incomplete SCI individuals

\begin{tabular}{lcccc}
\hline & \multicolumn{2}{c}{ Complete } & SCI lesion $(\mathrm{n}=8)$ & \multicolumn{2}{c}{ Incomplete SCI lesion $(\mathrm{n}=7)$} \\
Variable & Review 1 & Review 2 & Review 1 & Review 2 \\
\hline Time (months) & $10.1 \pm 0.7$ & $22.0 \pm 1.2$ & $9.7 \pm 0.7$ & $21.3 \pm 1.1$ \\
Duration (min/use) & $50.6 \pm 11.0$ & $80.6 \pm 23.5$ & $60.0 \pm 6.5$ & $79.3 \pm 27.9$ \\
Frequency (uses/week) & $3.4 \pm 0.7$ & $3.3 \pm 1.5$ & $2.7 \pm 0.8$ & $2.5 \pm 1.0$ \\
Total Intensity (min/week) & $142.5 \pm 21.8$ & $174.4 \pm 52.1$ & $158.6 \pm 47.5$ & $162.9 \pm 53.1$ \\
\hline
\end{tabular}


recoiled blind, replacing a shower curtain, cleaning the roof of a car and standing in a bar and at a party. The highest achievement using the Walkabout orthosis was by an individual with an incomplete $\mathrm{T} 9$ paraplegia and preserved proprioception in one leg who managed to walk her dogs in a park using forearm crutches. Improved standing stability was reported by all patients; however, few patients reported functional gains in the longer term. Indoor accessibility was not improved and was often hampered by the accompanying walking aid.

\section{Perceived benefits}

Benefits perceived by all patients who were reviewed twice whilst using the Walkabout are shown in Figure 2. No significant differences existed between individuals with complete and incomplete lesions. Physical and psychological benefits remained the most important. The most common physical benefit reported was maintenance of muscle length and joint range of motion. Others included reduced spasm and improved bowel management. Psychologically, two-thirds of patients in the first year described benefiting from being able to carry on conversation at eye level and experiencing their environment from a different perspective.

\section{Modifications and repairs}

The Walkabout device was relatively easy to fit and align, although it was found that few people were able to stand and balance well without individual orthotic customisation. All of the patients required adaptations, which included shoe raises, heel wedges, carbon fibre inserts, medial knee extensions and pads, bridging plates and in several patients lateral extension bars were fitted with a pelvic strap and an abdominal pad. Once set-up few required any adjustment, maintenance or repairs.

\section{Discontinued usage}

Five patients discontinued using the Walkabout between 7-20 months, three other patients were

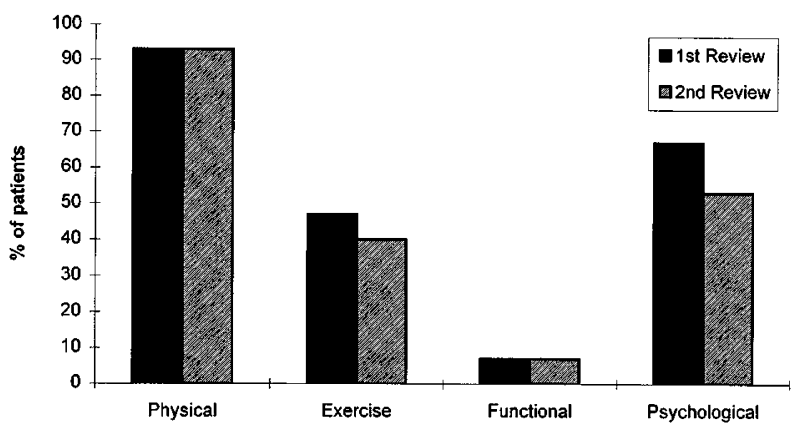

Figure 2 Perceived benefits reported at follow-up review unsuccessfully trained due to spinal immobility and another one was lost to follow-up. In four out of the five patients who have abandoned using the Walkabout, two with complete and two with incomplete lesions between $\mathrm{T} 4-\mathrm{T} 12$, the reason given was lack of functional enhancement over either pre-program orthoses (RGO, KAFO's) or the wheelchair. One patient's standing balance and function was compromised by ankle contractures. The remaining patient with a complete T10 paraplegia, although very proficient using a rollator frame and satisfied with the Walkabout, was forced to withdraw after 11 months due to persistent mechanical back pain related to previous multi-level laminectomy surgery.

Three individuals with complete paraplegia were unsuccessfully trained in the Walkabout orthosis due to very limited thoraco-lumbar spinal mobility. Thoraco-lumbar rigidity resulted in two individuals from posterior CD instrumentation from T11-L4 and T9- L2 respectively and in a third following T5-T11 posterior instrumentation despite removal secondary to marked extra-articular bone formation.

\section{Discussion}

The Walkabout device provides a new orthotic option to assist standing and short-distance mobility in paralysed individuals which differs significantly from all other currently available orthoses. The mediallymounted hinge joint linking two KAFO's was designed as an alternative to existing hip-knee-ankle-foot orthoses with the hope of improving some of the practical, functional and cosmetic difficulties experienced by SCI individuals. In particular, it was felt that the excessive bulkiness and unwieldiness of the $\mathrm{HGO}$ and LSU-RGO lead to awkward and tedious application and interfered with activities such as toileting and transfers. $^{22}$

Stallard et $a l^{25}$ highlighted several important areas of design compromise which affect the suitability of an orthotic device for meeting the needs of a particular individual. These were: (i) independence in application of the device, transfers and activities of daily living wearing the device such as toileting and negotiation of commonly encountered barriers such as kerbs; (ii) energy cost of ambulation; (iii) cosmesis, including style of ambulation in the orthosis, ability to disguise the orthosis under clothing, the type of associated walking aid and aesthetic design of the orthosis; (iv) system reliability/safety and (v) cost.

Clinical experience with the Walkabout orthosis has revealed the relative ease of fitting the device to KAFO's (with easy adjustment to change alignment), simplicity and robustness of design minimizing the need for ongoing adjustment or repairs and recyclability. The Walkabout device links semi-independent mechanisms into one structure by adding a hinge to KAFO's that joins the legs in an axis which is noncoincident with the hip joints. Force platform stabilometry has revealed a much less critical balance 
situation and significantly increased postural sway characteristics during activity, without need to maintain the body's centre of mass as closely regulated, in the Walkabout orthosis compared to KAFO's alone. ${ }^{26}$ Thus, the Walkabout orthosis allows users to stand for prolonged periods with good stability and far greater freedom and confidence to perform functional bimanual activities in standing.

However, the expected advantages over other orthoses were not clearly evident in several key areas of function, with difficulty still experienced when attaching and detaching the device under clothing for toileting, transferring wearing the Walkabout orthosis, particularly into and out of cars and negotiating inclines. Harvey et $a l^{27}$ also recently confirmed that while significantly more assistance was required to get to and from the standing position in the IsocentricRGO compared to the Walkabout orthosis, no significant differences in the level of assistance required and time taken were found between these orthoses with respect to ascending and descending stairs and kerbs or transferring into and out of cars.

Successful gait using the orthosis is dependent upon lateral flexion and weight shift to unweight the swing leg followed by anterior weight shifting to allow the free leg to swing through. A thoracic corset with crossed straps attached to the Walkabout provides a mechanical link between truncal movement and reciprocation. Cliquet et $a l^{24}$ demonstrated in a case study that medial linkage of bilateral KAFO's reduced energy cost of ambulation by $50 \%$ compared to conventional KAFO's alone, but no direct comparison with the RGO or HGO was made. ${ }^{24}$ Harvey et $a l^{27}$ have recently demonstrated a reduced speed of ambulation with a significant increase in energy cost in the Walkabout orthosis compared to the IsocentricRGO.

In this study, the most important factor preventing a successful outcome was found to be limitation of low thoracic and lumbar spinal mobility into lateral flexion. However, lack of mobility in the thoracolumbar region may not be a significant limiting factor in those individuals with active hip flexors or the ability to tilt the pelvis posteriorly. A relatively unaesthetic, wide-based gait pattern resulted from using the Walkabout and most complete paraplegic individuals lacked the confidence to ambulate at home outside of parallel bars when unaccompanied. Back pain relating to mechanics of the gait pattern may prove to be a limiting factor in some individuals. Great difficulty was found establishing a well balanced, comfortable standing position in those with contracture of hip and/or plantar flexors. Other factors adversely affecting outcome included excessive muscle spasm when standing and significant differences in muscle strength producing postural asymmetry in incomplete lesions. Marked lordosis in a mobile lumbar spine was compensated for by reversing the Walkabout and corset attachments to improve 'pelvic tuck' (see Figure 3). In those with poor control of the

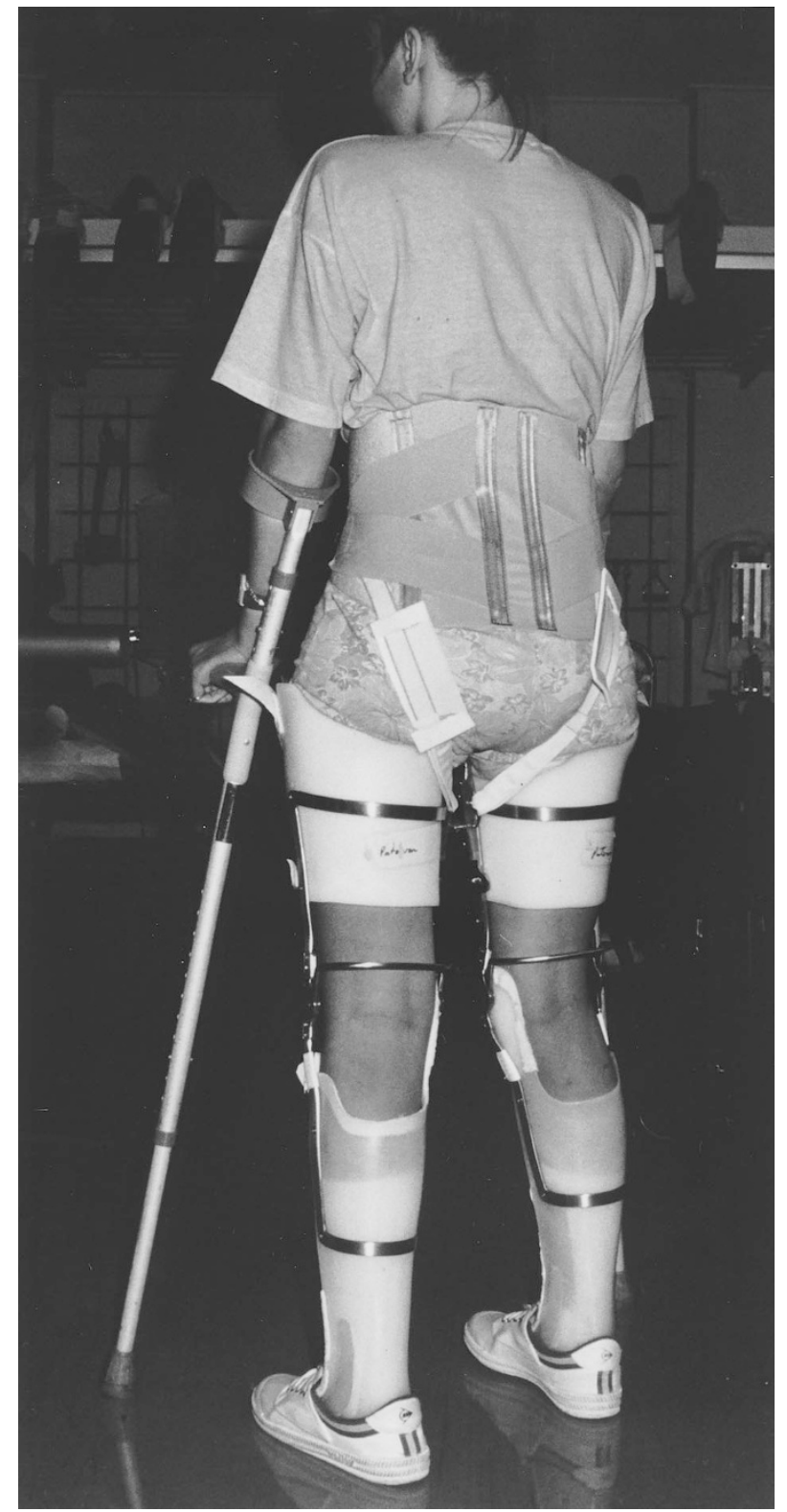

Figure 3 The Walkabout orthosis with corset attachment straps reversed and forearm crutches

lumbar lordosis (ie, high level paraplegics), further support was provided by side bars with pelvic and abdominal straps.

Despite a number of difficulties, many patients in this study have incorporated the Walkabout into their lifestyles, primarily for therapeutic rather than functional reasons, with physical and psychological benefits being identified as important. The demonstrated pattern of maintained usage by adult SCI individuals in the Walkabout orthosis, with a typical frequency of use between 1-3 times per week and total duration of usage between 2-3 hours per week, compares favourably with data previously published for the HGO. ${ }^{9}$ Longer term follow-up will be required 
to monitor continued usage. Not surprisingly, orthotic ambulatory ability and confidence were generally better in those where proprioception was preserved. However, more important for continuing usage than either level or degree of neurological impairment were personal motivation and commitment to initially develop and then maintain the acquired skills.

\section{Conclusion}

Selection criteria for the Walkabout orthosis should include patient commitment to assessment and training, control of muscle spasm, less than $5^{\circ}$ of hip or knee flexion contracture, a neutral ankle position achievable, pain-free mobility of thoraco-lumbar spine into lateral flexion, good upper limb strength, reasonable exercise tolerance and continuing patient motivation with realistic expectations. Each patient should be advised from the outset about the limitations of the orthosis, the time commitment and physical effort involved and realistic, achievable goals clearly defined to avoid disappointment.

This device is a useful orthosis to be considered particularly when assisting the paralysed patient to the standing position. It is not the ideal walking orthosis for continued use in spinal cord injured persons due to poor energy efficiency. In individuals with higher level, complete lesions the trunk support provided by the RGO and its improved efficiency would be of benefit. In certain situations in individuals with an incomplete lesion, modularity of the Walkabout orthosis allows it to be used during rehabilitation as an intermediate step in gait retraining, although the gait pattern developed may not be ideal.

\section{Acknowledgements}

The authors thank the AMP Society, the Orthotic Department of the Royal North Shore Hospital and Polymedic Pty Ltd for support which made this study possible. We also express special appreciation to Ms Frances Page-de Mars for her kind assistance in preparing this manuscript.

\section{References}

1 Abramson AS. Bone disturbances in injuries to the spinal cord and cauda equina (paraplegia): their prevention by ambulation. $J$ Bone Joint Surg 1948; 30A: $982-987$.

2 Hahn HR. Lower extremity bracing in paraplegics with usage follow-up. Paraplegia 1970; 8: $147-153$.

3 Guttmann L. Spinal cord injuries: Comprehensive management and research. Blackwell Scientific Publications, Oxford; 1973: 205

4 Kaplan PE. Gandhavadi B, Richards L, Goldschmidt J. Calcium balance in paraplegic patients: influence of injury duration and ambulation. Arch Phys Med Rehabil 1978; 59: 447-450.
5 Natvig H, McAdam R. Ambulation without wheelchairs for paraplegics with complete lesions. Paraplegia 1978; 16: $142-146$.

6 Kaplan PE et al. Reduction of hypercalciuria in tetraplegia after weight-bearing and strengthening exercises. Paraplegia 1981; 19: $289-293$.

7 Kunkel CF et al. Effect of 'standing' on spasticity, contracture, and osteoporosis in paralysed males. Arch Phys Med Rehabil 1993; 74: $73-78$.

8 Ogilvie C, Bowker P, Rowley DI. The physiological benefits of paraplegic orthotically aided walking. Paraplegia 1993; 31: $111-$ 115.

9 Summers BN, McClelland N, El Masri WS. A clinical review of the adult hip guidance orthosis (Parawalker) in traumatic paraplegics. Paraplegia 1988; 26: $19-26$.

10 Whittle MW et al. A comparative trial of two walking systems for paralysed people. Paraplegia 1991; 29: 97-102.

11 Heinemann A, Magiera-Planey R, Schiro-Geirst C, Gimenes G. Mobility for persons with spinal cord injury: an evaluation of two systems. Arch Phys Med Rehabil 1987; 68: 90 -93.

12 Rosman N, Spira E. Paraplegic use of walking braces: a survey. Arch Phys Med Rehabil 1974; 55: 310-314.

13 O'Daniel WE, Hahn HR. Follow-up usage of the Scott-Craig orthosis in paraplegia. Paraplegia 1981; 19: 373-378.

14 Marsolais EB, Kobetic R. Functional electrical stimulation for walking in paraplegia. J Bone Joint Surg 1987; 69 [AM]: $728-$ 733.

15 Solomonow M et al. The RGO generation II: muscle stimulation powered orthosis as a practical walking system for thoracic paraplegics. Orthopaedics 1989; 12: 1309-1315.

16 Isakov E, Douglas R, Berns P. Ambulation using the reciprocating gait orthosis and functional electrical stimulation. Paraplegia 1992; 30: 239 - 245.

17 Rose GK. Principles and practice of hip guidance articulations. Prosthetics and Orthotics International 1979; 3: 37-43.

18 Douglas R, Larson PF, D'Ambrosia R, McCall RE. The LSU reciprocating gait orthosis. Orthopaedics 1983; 6: $834-838$.

19 Kemp M. The advanced reciprocating gait orthosis. Proceedings of the National Assembly, American Orthotic and Prosthetic Association 1989.

20 Motloch W. Principles of orthotic management for child and adult paraplegia and clinical experience with the isocentric RGO. Proc 7 th World Congress ISPO June 28 - July 3, Chicago, Illinois, USA, 1992: 28 [Abstract].

21 Kirtley C, McKay SK. Total design of the 'Walkabout', a new paraplegic walking orthosis. Proc 7 th World Congress ISPO, June 28 - July 3, Chicago, USA, 1992: 39 [Abstract].

22 Kirtley C. Principles and practice of paraplegic locomotion: experience with the walkabout walking system. Aust Orth Pros Mag 1992; 7 (2): 4-8.

23 Cliquet A, Nene AV, Barnett R, Andrews BJ. FNS augmentation of reciprocating $\mathrm{HKAFO}$ and $\mathrm{KAFO}$ braces. Proc 2nd Int Workshop on F.E.S. Int. Soc. Artif, Organs, Vienna, 1986: 8386.

24 Cliquet A, Baxendale RH, Andrews BJ. Paraplegic locomotion and its metabolic energy expenditure, In: Rose FC, Jones R and Vrbova G, eds. Comprehensive Neurologic Rehabilitation 1989, Vol 3. Demos, USA: 139-146.

25 Stallard J, Major RE, Patrick JH. A review of the fundamental design problems of providing ambulation for paraplegic patients. Paraplegia 1989; 27: $70-75$.

26 Middleton J, Sinclair P. Postural stability and sway in the Walkabout orthosis. Proc Ann Sci Meeting Australasian Branch IMSOP, Christchurch, New Zealand, 1996: 57 [Abstract].

27 Harvey LA et al. A comparison of the Walkabout and the Isocentric Reciprocal Gait Orthosis in T9-12 paraplegics. Proc 35th Ann Sci Meeting IMSOP, Atlanta, Georgia, USA, 1996; 60 [Abstract]. 